\title{
Novel Intramolecular Reactivity of Oximes: Synthesis of Cyclic and Spiro-fused Imines
}

Cécile Savarin, "Christiane Grisé, Jerry Murry, Robert A. Reamer and Dave Hughes. Merck \& Co., Inc.; P.O. Box 2000, Rahway NJ 07065.

cecilesavarin@gmail.com

\section{Experimental}

\section{General Methods}

Reagents and solvents were obtained from commercial suppliers and were used without further purification or drying unless otherwise noted. Chromatography was done on silica gel (70-230 mesh). ${ }^{1} \mathrm{H}$ NMR and ${ }^{13} \mathrm{C}$ NMR spectra were recorded at 400, 500 or 600 and 101,126 or $151 \mathrm{MHz}$, respectively as stated in the text.

Solvents for extraction and chromatography were reagent grade and used as received. Dried solvents (THF, dioxane, toluene, DMF, DMAc) used as reaction media were purchased from Aldrich and titrated for water level prior to use with a Fisher Coulomatic K-F titrator $(\mathrm{KF}<300 \mu \mathrm{g} / \mathrm{mL})$. All manipulations were carried out under an inert atmosphere of nitrogen. Reagents were purchased from Aldrich Chemical Co. unless otherwise noted and were used without prior purification.

\section{General procedure for the preparation of oximes ${ }^{1}$ :}

To a solution of ketone (1.0 equiv.) in methanol $(45 \mathrm{~mL})$ was added hydroxylamine hydrochloride (2.0 equiv.) and sodium acetate ( 2.5 equiv.) sequentially. The heterogeneous solution was heated to $50{ }^{\circ} \mathrm{C}$ for $1-48$ hours (the reaction was slower if the substrate was sterically hindered: the reaction was followed by HPLC). The reaction mixture was cooled to room temperature. Water was added to crystallize the oxime. If crystals were not formed, the mixture was diluted in MTBE and washed with a saturated solution of sodium bicarbonate. The organic layer was then washed with brine, dried with $\mathrm{Na}_{2} \mathrm{SO}_{4}$, filtered and evaporated. The oximes were used without further purification. The reaction times and yields were not optimized.<smiles>C/C(=N\O)c1cc(Cl)ccc1C1CCN(C(=O)OCc2ccccc2)CC1</smiles> 
Benzyl 4-[4-chloro-2-( $N$-hydroxyethanimidoyl)phenyl]piperidine-1-carboxylate (1a). Following the general procedure, ketone $5 \mathbf{a}(9.67 \mathrm{~g}, 26 \mathrm{mmol})$, hydroxylamine hydrochloride ( $3.35 \mathrm{~g}, 52 \mathrm{mmol})$, sodium acetate $(5.33 \mathrm{~g}, 65 \mathrm{mmol})$ in methanol $(25 \mathrm{~mL})$ afforded 1a $(8.85 \mathrm{~g}, 88 \%)$ as a yellow solid: $\mathrm{mp}=123-129^{\circ} \mathrm{C}$. IR (neat, $\left.\mathrm{cm}^{-1}\right): 3327$ (b), 1684 (s), 1437 (s), 1217 (s). Major isomer component is described: ${ }^{1} \mathrm{H}$ NMR $\left(\mathrm{CDCl}_{3}\right.$, $400 \mathrm{MHz}$ ): $\delta 8.76$ (s, $1 \mathrm{H}), 7.40-7.33(\mathrm{~m}, 5 \mathrm{H}), 7.30(\mathrm{dd}, J=8.4,2.4 \mathrm{~Hz}, 1 \mathrm{H}), 7.20-7.16$ (m, 2H), 5.18 (s, 2H), 4.31 (br s, 2H), 2.89-2.78 (m, 3H), 2.22 (s, 3H), 1.76-1.73 (br m, $2 \mathrm{H}), 1.66-1.56(\mathrm{~m}, 2 \mathrm{H})$.

${ }^{13} \mathrm{C} \mathrm{NMR}\left(\mathrm{CDCl}_{3}, 101 \mathrm{MHz}\right): \delta 156.5,155.4,141.9,138.5,136.8,132.0,129.0,128.6$, 128.5, 128.1 (2C), 128.0, 67.3, 44.7, 38.5, 33.2, 16.6. Anal. Calcd for $\mathrm{C}_{21} \mathrm{H}_{23} \mathrm{ClN}_{2} \mathrm{O}_{3}$ : C, 65.20; H, 5.99; N, 7.24. Found: C, 65.04; H, 6.02; N, 7.07.<smiles>C/C(=N\O)c1ccccc1C1CCCCC1</smiles>

1-(2-Cyclohexylphenyl)ethanone oxime (1b). Following the general procedure, ketone 5b (3.69 g, $18.24 \mathrm{mmol})$, hydroxylamine hydrochloride $(2.35 \mathrm{~g}, 36.48 \mathrm{mmol})$, sodium acetate $(3.74 \mathrm{~g}, 45.60 \mathrm{mmol})$ in $20 \mathrm{~mL}$ methanol gave $1 \mathbf{b}(2.54 \mathrm{~g}, 64 \%)$ after crystallization from dichloromethane. Analytical sample was purified by flash chromatography eluting with $20 \%$ ethyl acetate $/ 80 \%$ hexane. MP $=149.8-154.7^{\circ} \mathrm{C}$. IR (neat, $\mathrm{cm}^{-1}$ ): 1651 (w), 1212 (s), $1153(\mathrm{~s}) .{ }^{1} \mathrm{H} \mathrm{NMR}\left(\mathrm{CDCl}_{3}, 400 \mathrm{MHz}\right): \delta 8.82(\mathrm{~s}, 1 \mathrm{H})$, 7.35-7.33 (m, 2H), 7.20-7.18 (m, 2H), 2.66 (tt, $J=11.6,2.8 \mathrm{~Hz}, 1 \mathrm{H}), 2.22(\mathrm{~s}, 3 \mathrm{H}), 1.86-$ $1.75(\mathrm{~m}, 5 \mathrm{H}), 1.48-1.24(\mathrm{~m}, 5 \mathrm{H}) .{ }^{13} \mathrm{C}$ NMR $\left(\mathrm{CDCl}_{3}, 101 \mathrm{MHz}\right): \delta 158.2,145.7,136.8$, 128.7, 128.3, 126.6, 125.7, 40.9, 34.6, 26.9, 26.2, 16.8. Anal. Calcd for $\mathrm{C}_{14} \mathrm{H}_{19} \mathrm{NO}$ : C, 77.38; H, 8.81; N, 6.45. Found: C, 77.17; H, 8.82; N, 6.32.<smiles>C/C(=N\O)c1ccccc1C1CCCC1</smiles>

1-(2-Cyclopentylphenyl)ethanone oxime (1c). Following the general procedure, ketone 5c $(2.06 \mathrm{~g}, 10.94 \mathrm{mmol})$, hydroxylamine hydrochloride $(1.41 \mathrm{~g}, 21.89 \mathrm{mmol})$ and sodium acetate $(2.24 \mathrm{~g}, 27.35 \mathrm{mmol})$ in $12 \mathrm{~mL}$ methanol yielded $1 \mathrm{c}(1.33 \mathrm{~g}, 60 \%)$ as yellow solid mp: 107-109 ${ }^{\circ} \mathrm{C}$. IR (neat, $\mathrm{cm}^{-1}$ ): 3240 (b), 1361 (w), 1208 (m), 1151 (m) ${ }^{1} \mathrm{H}$ NMR $\left(\mathrm{CDCl}_{3}, 400 \mathrm{MHz}\right): \delta 9.11(\mathrm{~s}, 1 \mathrm{H}), 7.36-7.34(\mathrm{~m}, 2 \mathrm{H}), 7.20-7.18(\mathrm{~m}, 2 \mathrm{H}), 3.12(\mathrm{~m}, 1 \mathrm{H})$, $2.23(\mathrm{~s}, 3 \mathrm{H}), 2.12-2.01(\mathrm{~m}, 2 \mathrm{H}), 1.89-1.81(\mathrm{~m}, 2 \mathrm{H}), 1.70-1.58(\mathrm{~m}, 4 \mathrm{H}) .{ }^{13} \mathrm{C} \mathrm{NMR}\left(\mathrm{CDCl}_{3}\right.$, $101 \mathrm{MHz}): \delta 158.4,144.8,137.5,128.9,128.1,126.6,125.6,42.2,35.6,26.1,16.9$. HRMS 203.13 for $\mathrm{C}_{13} \mathrm{H}_{17} \mathrm{NO}$ (Calc. [M+H] 204.1383; meas. [M+H] 204.1415). 
<smiles>CC(C)c1ccccc1/C(=N/O)C(F)(F)F</smiles>

2,2,2-Trifluoro-1-(2-isopropylphenyl)ethanone oxime (1d). Following the general

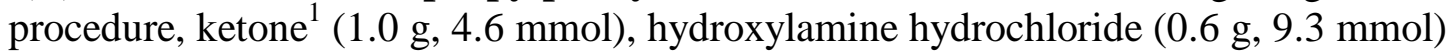
and sodium acetate $(0.9 \mathrm{~g}, 11.6 \mathrm{mmol})$ in $7 \mathrm{~mL}$ methanol yielded $1 \mathrm{~d}(1.3 \mathrm{~g}, 100 \%)$ as a white solid mp: 57.3-61.0 ${ }^{\circ} \mathrm{C}$. IR (neat, $\mathrm{cm}^{-1}$ ): 1655 (w), 1557 (w), 1216 (s), 1153 (s). Compound exists as a 1:1 mixture of syn/anti oxime isomers-both isomers are reported together. ${ }^{1} \mathrm{H} \mathrm{NMR}\left(\mathrm{CDCl}_{3}, 400 \mathrm{MHz}\right): \delta 8.53(\mathrm{~s}, 1 \mathrm{H}), 8.24(\mathrm{~s}, 1 \mathrm{H}), 7.51-7.39(\mathrm{~m}, 4 \mathrm{H})$, 7.32-7.14 (m, 4H), 2.95 (septet, $J=6.8 \mathrm{~Hz}, 1 \mathrm{H}$ ), 2.76 (septet, $J=6.8 \mathrm{~Hz}, 1 \mathrm{H}$ ), 1.27-1.21 $(\mathrm{m}, 12 \mathrm{H}) .{ }^{13} \mathrm{C} \mathrm{NMR}\left(\mathrm{CDCl}_{3}, 101 \mathrm{MHz}\right): \delta 149.4(\mathrm{q}, J=31.3 \mathrm{~Hz}), 148.9(\mathrm{q}, J=32.1 \mathrm{~Hz})$, 148.7, 148.3, 130.8, 130.6, 129.6, 128.3, 127.7, 126.14, 126.08, 126.0, 125.9, 125.17, 120.5 (q, $J=274.7 \mathrm{~Hz}), 118.1$ (q, $J=282.7 \mathrm{~Hz}), 31.6,31.0,24.1,24.0,23.9$. Anal. Calcd for $\mathrm{C}_{11} \mathrm{H}_{12} \mathrm{~F}_{3} \mathrm{NO}$ : C, 57.14; H, 5.23; N, 6.06. Found: C, 57.32; H, 5.18; N, 6.13.

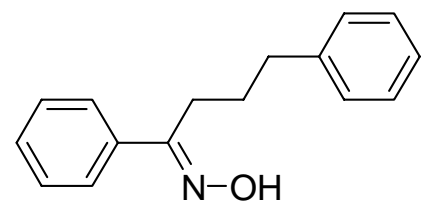

1,4-Diphenylbutan-1-one oxime (1e). ${ }^{2}$ Following the general procedure, ketone ${ }^{1}(5 \mathrm{~g}$, $22.0 \mathrm{mmol})$, hydroxylamine hydrochloride $(2.9 \mathrm{~g}, 42.2 \mathrm{mmol})$ and sodium acetate $(3 \mathrm{~g}$, $36.0 \mathrm{mmol})$ in $80 \mathrm{~mL}$ methanol yielded 1e $(5.3 \mathrm{~g}, 99 \%)$ as a white solid after aqueous work-up. Mp: $103-104{ }^{\circ} \mathrm{C} .{ }^{1} \mathrm{H}$ NMR (DMSO-d 6 , $400 \mathrm{MHz}$ ): $\delta 11.13$ (s, 0.6H), 7.59-7.57 $(\mathrm{m}, 2 \mathrm{H}), 7.39-7.34(\mathrm{~m}, 3 \mathrm{H}), 7.27-7.24(\mathrm{~m}, 2 \mathrm{H}), 7.17-7.14(\mathrm{~m}, 3 \mathrm{H}), 2.75-2.71(\mathrm{~m}, 2 \mathrm{H})$, $2.62(\mathrm{t}, J=7.6 \mathrm{~Hz}, 2 \mathrm{H}), 1.77-1.69(\mathrm{~m}, 2 \mathrm{H}) .{ }^{13} \mathrm{C} \mathrm{NMR}$ (DMSO-d $\left.6,101 \mathrm{MHz}\right): \delta 156.9$, 142.2, 136.5, 129.0, 128.9 (2C0, 128.7 (4C), 126.2 (3C), 35.7, 28.2, 25.2. HRMS 203.13 for $\mathrm{C}_{16} \mathrm{H}_{17} \mathrm{NO}$ (Calc. [M+H] 240.1383; meas. [M+H] 240.1409).

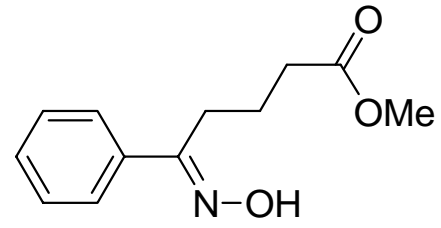

5-Hydroxyimino-5-phenylpentanoic acid methyl ester (1f). ${ }^{3}$ Following the general

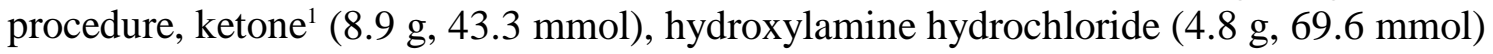
and sodium acetate $(5.1 \mathrm{~g}, 62.2 \mathrm{mmol})$ in $90 \mathrm{~mL}$ methanol yielded $\mathbf{1 f}(8.6 \mathrm{~g}, 90 \%)$ as an

\footnotetext{
${ }^{1}$ Ketone commercially available

2 (a) Stoermer, S.; Chem. Ber. 1951, 1728. (b) Ono, K.; Kawakami, H.; Katsube, J. Eur. Pat. Appl. (1980), EP 1721719801015.

${ }^{3}$ (a) Jong, L.; Zaveri, N.; Toll, L. Bioorg. Med. Chem. Lett. 2004, 14, 181. (b) Imoto, H.; Sugiyama, Y.; Kimura, H.; Momose, Y. Chem. Pharm. Bull. 2003, 51, 138.
} 
oil after aqueous work-up. ${ }^{1} \mathrm{H}$ NMR $\left(\mathrm{CDCl}_{3}, 400 \mathrm{MHz}\right): \delta 9.55$ (br s, $\left.1 \mathrm{H}\right), 7.67-7.63(\mathrm{~m}$, 2H), 7.48-7.37 (m, 3H), $3.67(\mathrm{~s}, 3 \mathrm{H}), 2.91-2.89(\mathrm{~m}, 2 \mathrm{H}), 2.41(\mathrm{t}, J=7.2 \mathrm{~Hz}, 2 \mathrm{H}), 1.94$ (pent, $J=7.2 \mathrm{~Hz}, 2 \mathrm{H}$ ). HRMS 221.11 for $\mathrm{C}_{12} \mathrm{H}_{15} \mathrm{NO}_{3}$ (Calc. [M+H] 222.1125; meas. $[\mathrm{M}+\mathrm{H}] 222.1121,1.8$ ppm error).

\section{$\underline{\text { Representative procedure for cyclization of oxime(Heat method): }}$}

In a 3-neck flask, oxime (1.2 mmol, 1.00 equiv.) was degassed under nitrogen and dissolved in dimethylacetamide $(12 \mathrm{~mL})$. Acetic anhydride $(1.3 \mathrm{mmol}, 1.05$ equiv.) was added to the reaction mixture. The solution was stirred at $135{ }^{\circ} \mathrm{C}$ until completion of the reaction (reaction followed by HPLC). Once the acetate completely disappeared, the reaction was cooled to room temperature. The mixture was diluted in MTBE and washed two times with a saturated solution of sodium bicarbonate. The organic layer was washed with brine, dried with $\mathrm{Na}_{2} \mathrm{SO}_{4}$, filtered and evaporated. Purification by flash chromatography afforded clean product.

(Microwave method) : In a microwave tube, oxime ( $0.83 \mathrm{mmol}, 1.00$ equiv.) was degassed under nitrogen and dissolved in dimethylacetamide $(3 \mathrm{~mL})$. Acetic anhydride (0.87 mmol, $1.05 \mathrm{eq})$ was added to the reaction mixture. The solution was stirred at room temperature for one hour. A seal was put on the microwave tube. The reaction was performed in a SmithSynthesizer Robot Microwave for $17-30 \mathrm{~min}$ at $240{ }^{\circ} \mathrm{C}$. The reaction was followed by HPLC. If the reaction was not complete, it was submitted to microwave conditions for another $30 \mathrm{~min}$ at the same temperature. The workup and purification were the same as the heat method.<smiles>CC1=NC2(CCN(C(=O)OCc3ccccc3)CC2)c2ccc(Cl)cc21</smiles>

Benzyl 5-chloro-3-methyl-1' $H$-spiro[iso-indole-1,4'-piperidine]-1'-carboxylate (3a). Following the general heating method, oxime 1a $(478 \mathrm{mg}, 1.2 \mathrm{mmol})$ and acetic anhydride $(122 \mu \mathrm{l}, 1.3 \mathrm{mmol})$ in dimethylacetamide $(12 \mathrm{~mL})$ yielded the spiro compound 3a as an oil. Purification by flash chromatography (50\% ethyl acetate/ $50 \%$ hexane) gave $372 \mathrm{~g}$ of the spiro as an oil $\left(82 \%\right.$ yield). IR (neat, $\left.\mathrm{cm}^{-1}\right): 1695(\mathrm{~s}), 1422(\mathrm{~m}), 1235$ (s), 1206 (s). ${ }^{1} \mathrm{H} \mathrm{NMR}\left(\mathrm{CDCl}_{3}, 400 \mathrm{MHz}\right): \delta 7.47$ (d, $\left.J=2.0 \mathrm{~Hz}, 1 \mathrm{H}\right), 7.43-7.34(\mathrm{~m}, 6 \mathrm{H})$, $7.31(\mathrm{~d}, J=8.0 \mathrm{~Hz}, 1 \mathrm{H}), 5.21$ (s, 2H), 4.30-4.15 (br m, 2H), 3.65-3.51 (br m, 2H), 2.46

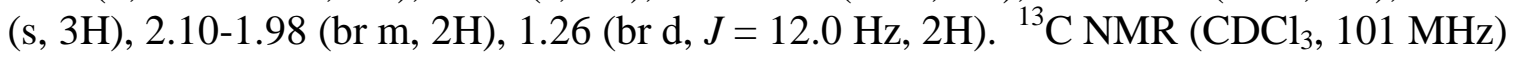
: $\delta 167.4,155.8,155.6,140.3,137.0,133.8,128.8,128.6,128.1,128.0,122.1,121.7$, 75.0, 67.2, 41.9, 34.5, 16.6. ${ }^{15} \mathrm{~N} \mathrm{NMR}\left(\mathrm{CDCl}_{3}, 60 \mathrm{MHz}\right): \delta 332$; chemical shift obtained from ${ }^{1} \mathrm{H}^{15} \mathrm{~N}$ HMBC. HRMS 368.1292 for $\mathrm{C}_{21} \mathrm{H}_{21} \mathrm{ClN}_{2} \mathrm{O}_{2}$ (Calc. [M+Na] 391.1184; meas. [M+Na] 391.1225). 
<smiles>CC1=NC2(CCCCC2)c2ccccc21</smiles>

3'-Methylspiro[cyclohexane-1,1'-iso-indole] (3b). Following the general heating method, oxime $\mathbf{1 b}(230 \mathrm{mg}, 1.1 \mathrm{mmol})$ and acetic anhydride (104 $\mu \mathrm{l}, 1.1 \mathrm{mmol})$ in dimethylacetamide $(10 \mathrm{~mL})$ yielded the spiro compound $\mathbf{3 b}$. Purification by flash chromatography (20\% ethyl acetate/ $80 \%$ hexane) gave $163 \mathrm{mg}$ of the spiro as a solid (78\% yield). MP: $51-53{ }^{\circ} \mathrm{C}$. IR (neat, $\mathrm{cm}^{-1}$ ): $1608(\mathrm{w}), 1378(\mathrm{w}), 1208(\mathrm{~s}), 1152(\mathrm{~s}) .{ }^{1} \mathrm{H}$ NMR $\left(\mathrm{CDCl}_{3}, 400 \mathrm{MHz}\right): \delta$ 7.50-7.46 (m, 2H), 7.36-7.34 (m, 2H), $2.47(\mathrm{~s}, 3 \mathrm{H}), 2.06-$ $1.92(\mathrm{~m}, 2 \mathrm{H}), 1.83-1.70(\mathrm{~m}, 5 \mathrm{H}), 1.61-1.52(\mathrm{~m}, 1 \mathrm{H}), 1.48-1.45(\mathrm{~m}, 2 \mathrm{H}) .{ }^{13} \mathrm{C} \mathrm{NMR}$ $\left(\mathrm{CDCl}_{3}, 101 \mathrm{MHz}\right): \delta 167.2,159.2,138.7,128.1,127.2,121.6,121.2,77.1,35.2,25.9$, 23.5, 16.6. Anal. Calcd for $\mathrm{C}_{14} \mathrm{H}_{17} \mathrm{~N}$ : C, 84.37; H, 8.60; N, 7.03. Found: $\mathrm{C}_{14} \mathrm{H}_{17} \mathrm{~N}$ : C, 84.49; H, 8.72; N, 7.05.<smiles>CC1=NC2(CCCC2)c2ccccc21</smiles>

3'-Methylspiro[cyclopentane-1,1'-iso-indole] (3c). Following the general microwave method, oxime 1c $(228 \mathrm{mg}, 1.1 \mathrm{mmol})$ and acetic anhydride $(111 \mu \mathrm{l}, 1.2 \mathrm{mmol})$ in dimethylacetamide $(3 \mathrm{~mL})$ yielded the spiro compound 3c. Purification by flash chromatography (30\% ethyl acetate/ $70 \%$ hexane) gave $141 \mathrm{~g}$ of the spiro as an oil (68 $\%$ yield) IR (neat, $\mathrm{cm}^{-1}$ ): $1610(\mathrm{w}), 1380(\mathrm{~m}), 1210$ (s), 1152 (s). ${ }^{1} \mathrm{H} \mathrm{NMR}\left(\mathrm{CDCl}_{3}, 400\right.$ MHz): $\delta$ 7.44-7.35 (m, 4H), 2.44 (s, 3H), 2.30-2.13 (m, 2H), 2.13-1.94 (m, 4H), 1.94$1.83(\mathrm{~m}, 2 \mathrm{H}) .{ }^{13} \mathrm{C} \mathrm{NMR}\left(\mathrm{CDCl}_{3}, 101 \mathrm{MHz}\right): \delta 166.8,157.6,138.8,128.5,127.0,120.9$, $120.8,84.7,37.2,26.1,16.5$.

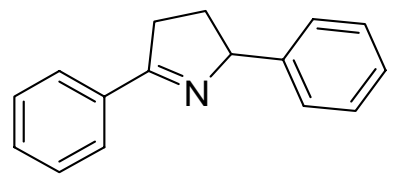

\section{Dehydropyrrolidine (3e). ${ }^{4}$}

Following the general heating method (microwave, $240{ }^{\circ} \mathrm{C}, 20 \mathrm{~min}$ ), oxime 1e (98 $\mathrm{mg}, 0.4 \mathrm{mmol})$ and acetic anhydride $(40 \mu \mathrm{l}, 0.4 \mathrm{mmol})$ in dimethylacetamide $(0.5 \mathrm{~mL})$ yielded the spiro compound 3e. Purification by flash chromatography (25\% ethyl acetate/ $75 \%$ hexane) gave $56 \mathrm{mg}$ of the spiro as a solid (62\% yield). ${ }^{1} \mathrm{H} \mathrm{NMR}\left(\mathrm{CDCl}_{3}\right.$, $600 \mathrm{MHz}): \delta 7.98-7.96(\mathrm{~m}, 2 \mathrm{H}), 7.49-7.43(\mathrm{~m}, 3 \mathrm{H}), 7.37-7.32(\mathrm{~m}, 4 \mathrm{H}), 7.28-7.25(\mathrm{~m}$, $1 \mathrm{H}), 5.34$ (br t, $J=7.6 \mathrm{~Hz}, 1 \mathrm{H}), 3.19$ (dddd, $J=16.2,9.4,4.2,1.9 \mathrm{~Hz}, 1 \mathrm{H}), 3.06-3.00$ (m, $1 \mathrm{H}), 2.65-2.59(\mathrm{~m}, 1 \mathrm{H}), 1.96-1.90(\mathrm{~m}, 1 \mathrm{H}) .{ }^{13} \mathrm{C} \mathrm{NMR}\left(\mathrm{CDCl}_{3}, 151 \mathrm{MHz}\right): \delta 173.9$, 144.8, 134.6, 130.9, 128.7 (4C), 128.1 (2C), 127.0, 126.8 (2C), 76.2, 35.8, 32.7.

\footnotetext{
${ }^{4}$ Auricchio, S.; Grassi, S.; Malpezzi, L.; Sartori, A.S.; Truscello, A.M. Eur. J. Org. Chem. 2001; 6; 1183.
} 
<smiles>COC(=O)C1CCC(c2ccccc2)=N1</smiles>

\section{Methyl-5-phenyl-3,4-dihydro-2H-pyrrole-2-carboxylate (3f). ${ }^{5}$}

Following the general heating method, oxime 1f $(201 \mathrm{mg}, 0.9 \mathrm{mmol})$ and acetic anhydride $(85 \mu \mathrm{l}, 0.9 \mathrm{mmol})$ in dimethylacetamide $(7 \mathrm{~mL})$ yielded the spiro compound 3f. Purification by flash chromatography (ethyl acetate:hexanes, 3:7) gave $112 \mathrm{mg}$ of the spiro as an oil (61\% yield). ${ }^{1} \mathrm{H} \mathrm{NMR}\left(\mathrm{CDCl}_{3}, 400 \mathrm{MHz}\right): \delta 7.91-7.88(\mathrm{~m}, 2 \mathrm{H}), 7.48-7.40$ $(\mathrm{m}, 3 \mathrm{H}), 4.96-4.91(\mathrm{~m}, 1 \mathrm{H}), 3.80(\mathrm{~s}, 3 \mathrm{H}), 3.23-3.14(\mathrm{~m}, 1 \mathrm{H}), 3.05-2.96(\mathrm{~m}, 1 \mathrm{H}), 2.42-$ $2.23(\mathrm{~m}, 2 \mathrm{H})$.<smiles>CC(=O)Nc1ccccc1C1CCCCC1</smiles>

\section{$N$-(2-Cyclohexylphenyl)acetamide (4b). ${ }^{6}$}

Sample is a 3.5:1 mixture of amide rotamers - the major rotamer is reported at $0{ }^{\circ} \mathrm{C}$. ${ }^{1} \mathrm{H} \mathrm{NMR}\left(\mathrm{CDCl}_{3}, 500 \mathrm{MHz}\right): \delta$ 7.56-7.54 (m, 1H), 7.29-7.27 (m, 1H), 7.21-7.19 (m, 2H), 7.18 (br s, 1H), 2.59 (tt, $J=11.5,2.8 \mathrm{~Hz}, 1 \mathrm{H}), 2.22(\mathrm{~s}, 3 \mathrm{H}), 1.87-1.70(\mathrm{~m}, 6 \mathrm{H}), 1.46-1.34$ $(\mathrm{m}, 4 \mathrm{H}) .{ }^{13} \mathrm{C} \mathrm{NMR}\left(\mathrm{CDCl}_{3}, 126 \mathrm{MHz}\right): \delta 169.3,140.6,133.9,126.6,126.5,126.5$, $125.7,38.8,33.6,27.1,26.2,24.4$.<smiles>CC(=O)c1cc(Cl)ccc1C1CCN(C(=O)OCc2ccccc2)CC1</smiles>

4-(2-Acetyl-4-chlorophenyl)-piperidine-1-carboxylic acid benzyl ester (5a).

Ketone was prepared from the corresponding nitrile and MeLi prepared as described in the literature. ${ }^{7} \mathrm{MP}: 81-83^{\circ} \mathrm{C}$. IR (neat, $\left.\mathrm{cm}^{-1}\right): 1695(\mathrm{~s}), 1685(\mathrm{~s}) .{ }^{1} \mathrm{H}$ NMR $\left(\mathrm{CDCl}_{3}, 400 \mathrm{MHz}\right): \delta 7.54(\mathrm{~d}, J=2.2 \mathrm{~Hz}, 1 \mathrm{H}), 7.43-7.25(\mathrm{~m}, 6 \mathrm{H}), 7.28(\mathrm{~d}, J=8.4 \mathrm{~Hz}$, $1 \mathrm{H}), 5.16(\mathrm{~s}, 2 \mathrm{H}), 4.32$ (br s, $2 \mathrm{H}), 3.27(\mathrm{tt}, J=12.0,3.3 \mathrm{~Hz}, 1 \mathrm{H}), 2.94-2.82(\mathrm{~m}, 2 \mathrm{H}), 2.59$ $(\mathrm{s}, 3 \mathrm{H}), 1.80(\mathrm{~d}, J=12.8 \mathrm{~Hz}, 2 \mathrm{H}), 1.69-1.51(\mathrm{~m}, 2 \mathrm{H}) .{ }^{13} \mathrm{C} \mathrm{NMR}\left(\mathrm{CDCl}_{3}, 101 \mathrm{MHz}\right)$ :

\footnotetext{
5 (a) Mkairi, A.; Hamelin, J. Tetrahedron Lett. 1987, 28, 1397. (b) Lakhlifi, T.; Sedqui, A.; Fathi, T.; Laude, B.; Robert, J.-F. Can. J. Chem. 1994; 72; 1417.

${ }^{6}$ Hickinbottom, W.J. J. Chem. Soc. 1932; 2646.

${ }^{7}$ Boice, G.N.; Savarin, C.G.; Murry, J.A.; Conrad, K.; Matty, L.; Corley, E.; Smitrovich, J.; Hughes, D. Tetrahedron. 2004, 60, 11367.
} 
$\delta$ 201.5, 155.3, 143.3, 139.9, 137.0, 131.9, 131.4, 128.8, 128.6, 128.4, 128.0, 128.0, 67.1, 44.7, 37.7, 33.2, 30.4. Anal. Calcd for $\mathrm{C}_{21} \mathrm{H}_{22} \mathrm{ClNO}_{3}: \mathrm{C}, 67.83 ; \mathrm{H}, 5.96 ; \mathrm{N}, 3.77$. Found: C, 67.84; H, 5.93; N, 3.83.

\section{General Protocol for Ketone Formation ${ }^{8}$}

To a solution of bromoacetophenone (1 equiv.) in dimethylacetamide was added $\mathrm{Cl}_{2} \mathrm{Pd}(\mathrm{dppf})(2 \mathrm{~mol} \%)$ and $\mathrm{CuI}(3.5 \mathrm{~mol} \%)$ at once. Aliphatic zinc halide (1.25 equiv.) was cannulated into the reaction mixture over 20 minutes. The solution was stirred at 60 ${ }^{\circ} \mathrm{C}$ for 2-7 hours (Followed reaction by HPLC). After the reaction was cooled to room temperature, the reaction mixture was filtered through celite and washed with ethyl acetate. The solution was extracted two times with $1 \mathrm{~N} \mathrm{HCl}$. The organic layer was then washed with brine, dried with sodium sulfate, filtered and evaporated. Eluting through a plug of silica purified the mixture. The ketone was used in the next step without further purification.<smiles>CC(=O)c1ccccc1C1CCCCC1</smiles>

1-(2-cyclohexylphenyl)ethanone (5b) : Following the general procedure for the Negishi cross-coupling, bromoacetophenone (4.02 g, $0.020 \mathrm{~mol})$, cyclohexyl zinc bromide (0.5 M in THF, $50 \mathrm{~mL}, 0.025 \mathrm{~mol}), \mathrm{Cl}_{2} \mathrm{Pd}(\mathrm{dppf})(309 \mathrm{mg}, 0.0004 \mathrm{~mol})$ and $\mathrm{CuI}$ $(150 \mathrm{mg}, 0.0008 \mathrm{~mol})$ in dimethylacetamide $(10 \mathrm{~mL})$ gave the desired ketone as an oil in $91 \%$ yield $(3.69 \mathrm{~g})$. Purification of an analytical sample was done by flash chromatography (20\% ethyl acetate/ $80 \%$ hexane) IR (neat, $\left.\mathrm{cm}^{-1}\right): 1686(\mathrm{~s}), 1447(\mathrm{~m})$,

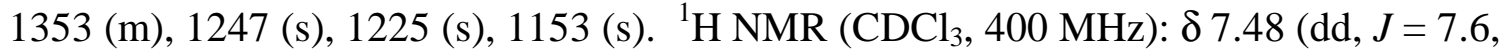
$0.8 \mathrm{~Hz}, 1 \mathrm{H}), 7.41-7.40(\mathrm{~m}, 2 \mathrm{H}), 7.24-7.20(\mathrm{~m}, 1 \mathrm{H}), 3.06(\mathrm{tt}, J=11.7,2.8 \mathrm{~Hz}, 1 \mathrm{H}), 2.57$ $(\mathrm{s}, 3 \mathrm{H}), 1.86-1.75(\mathrm{~m}, 5 \mathrm{H}), 1.46-1.40(\mathrm{~m}, 4 \mathrm{H}), 1.30-1.21(\mathrm{~m}, 1 \mathrm{H}) .{ }^{13} \mathrm{C} \mathrm{NMR}\left(\mathrm{CDCl}_{3}, 100\right.$ $\mathrm{MHz}): \delta 203.7,146.7,139.2,130.9,127.7,127.2,125.4,40.0,34.6,30.8,27.0,26.3$.<smiles>CC(=O)c1ccccc1C1CCCC1</smiles>

1-(2-Cyclopentylphenyl)ethanone (5c) : Following the general procedure for the Negishi cross-coupling, bromoacetophenone (4.0 g, $0.020 \mathrm{~mol})$, cyclopentyl zinc bromide (0.5 M in THF, $50 \mathrm{~mL}, 0.025 \mathrm{~mol}), \mathrm{Cl}_{2} \mathrm{Pd}(\mathrm{dppf})(328 \mathrm{mg}, 0.0004 \mathrm{~mol})$ and $\mathrm{CuI}(133 \mathrm{mg}$,

${ }^{8}$ Corley, E.; Conrad, K.; Murry, J.; Savarin, C.; Holko, J.; Boice, G. J. Org. Chem. 2004, 69, 5120. 
$0.0007 \mathrm{~mol})$ in dimethylacetamide $(10 \mathrm{~mL})$ gave the desired ketone as an oil in $88 \%$ yield (3.30 g). Purification of an analytical sample was done by flash chromatography (2.5\% ethyl acetate/ $97.5 \%$ hexane). IR (neat, $\mathrm{cm}^{-1}$ ): 1685 (s), 1246 (s), 1224 (s), 1152 (s). ${ }^{1} \mathrm{H} \mathrm{NMR}\left(\mathrm{CDCl}_{3}, 400 \mathrm{MHz}\right): \delta$ 7.47-7.45 (m, 1H), 7.42-7.40 (m, 2H), 7.24-7.20 (m, $1 \mathrm{H}), 3.52-3.43(\mathrm{~m}, 1 \mathrm{H}), 2.58(\mathrm{~s}, 3 \mathrm{H}), 2.09-2.07(\mathrm{~m}, 2 \mathrm{H}), 1.83-1.80(\mathrm{~m}, 2 \mathrm{H}), 1.72-1.70$ $(\mathrm{m}, 2 \mathrm{H}), 1.69-1.57(\mathrm{~m}, 2 \mathrm{H}) .{ }^{13} \mathrm{C} \mathrm{NMR}\left(\mathrm{CDCl}_{3}, 100 \mathrm{MHz}\right): \delta 203.9,145.3,140.0,130.9$, 127.4, 127.1, 125.3, 41.6, 35.2, 30.7, 25.9. HRMS 188.12 for $\mathrm{C}_{13} \mathrm{H}_{16} \mathrm{O}(\mathrm{Calc} . \mathrm{M}+\mathrm{H}]$ 189.1273; meas. [M+H] 189.1269). 